\title{
Computer simulation study of polydispersity effect on the phase behavior of short diblock copolymers
}

\author{
Gui-Sheng Jiao, ${ }^{1}$ Yue Li, ${ }^{1}$ Hu-Jun Qian, ${ }^{1, \text { a) }}$ and Zhong-Yuan Lu ${ }^{1}$ \\ State Key Laboratory of Supramolecular Structure and Materials, Institute of Theoretical Chemistry, Jilin University, \\ Changchun 130023, China
}

(Dated: 2 April 2016)

Recent progress in producing short chain length diblock copolymers facilitates the fabrication of block copolymer nanostructures with extremely small feature sizes. In this work, phase diagrams for monodisperse, onesided and two-sided polydisperse short diblock copolymer melts are constructed using dissipative particle dynamics (DPD) simulations. Detailed comparisons are carried out between these systems to elucidate the influence of chain length polydispersity on the phase behavior of short diblock copolymers. In particular, we find an unexpected stability of a bicontinuous structure over a wide composition range between $0.7<f \leq 0.8$ in one-sided polydisperse system, where $f$ is the volume fraction of the polydisperse component. In the case of the two-sided polydisperse system, due to the presence of highly asymmetrical block copolymers and the pulling-away of short blocks of these molecules into opposite domain, an up-shift is found in the order-disorder transition boundary to larger $\chi N$ values over the composition range we investigated.

Keywords: short diblock copolymers, polydispersity, computer simulation

\section{INTRODUCTION}

Due to the intrinsic mutual incompatibility between distinct blocks, block copolymers (BCPs) have an intrinsic capability of forming novel ordered structures ${ }^{1,2}$. Many investigations have shown that these ordered structures and their formation processes can be influenced by many factors, for instance, a competing length scale effect imposed by different internal molecular architectures, ${ }^{3,4}$ or spatial confinement effects caused in spherical $^{5}$ or cylindrical ${ }^{6}$ spaces. It can be also directed by topographical templates. ${ }^{7}$ Despite these complex influence factors, recent developments in the field also show that the chain length of the BCPs is also very important, especially in the case of short chain length systems. From a practical point of view, an important practical application of these BCP ordered structures is in the filed of modern nanotechnology where these structures are being demanded. For instance, lamella is a common structure utilized for nanolithography, while gyroid structure favoring exciton or ion transportations are often used for fabrication of photovoltaic films in solar cells or separator membranes in batteries. ${ }^{8}$ Conventionally, synthetic diblock copolymers, which can form ordered structures, usually have very long chain lengths $(N)$ and therefore have large enough $\chi N$ values exceeding the microphase separation threshold, $(\chi N)_{\text {ODT }}=10.495$. However, with the development of nanotechnology, ordered structures with smaller dimensions are demandingly required. ${ }^{9}$ Recent progress in synthesizing short diblock copolymers ${ }^{10,11}$ with strong segregation strength (small $N$, large $\chi$ ) enables the fabrication of sub-10 nm sized patterns, making block copolymer nanolithography a potential priori to traditional photolithography. Most

a) Electronic mail: hjqian@jlu.edu.cn importantly, it offers a possibility of breaking down the bottle-neck of shrinking the domain size of integrated circuits, which is extremely important to fabricate more faster, cheaper and energy-efficient microchips. Therefore, it's practically very important to understand the phase behavior of short diblock copolymers.

On the other hand, understanding the phase behavior of short block copolymers ${ }^{12}$ are also very important from a fundamental point of view since Leibler's meanfield theory is only applicable to infinite- $N$ systems with a weak segregation assumption at ODT boundary. Even with correction developed by Fredrickson and Helfand $(\mathrm{FH}),{ }^{13}$ it is still only strictly valid with a very long chain length $\left(N \sim 10^{9}\right)$. A recent work by Morse and coworkers $^{14}$ showed that competing phases near the ODT boundary become strongly segregated in short (low- $N$ ) diblock copolymer system and violate the weak segregation assumption, therefore resulting a stronger correction term in $(\chi N)_{\text {ODT }}$. In practice, a good comparison of the experiment and simulation results to the theory needs an effective estimation of $\chi$ parameter. However, conventional way of fitting $\chi(T)$ relation according to experimentally observed $T_{\text {ODT }}$ data based on mean-field theory prediction of $(\chi N)_{\text {ODT }}=10.495$ neglects the consequences may caused by finite chain length $N$. A more sophisticated way is to determine the $\chi$ parameter by fitting results for structure factor $S(q)$ in the disordered phase to predictions of the renormalized one-loop (ROL) theory developed by Morse and coworkers. ${ }^{14}$ Using this new fitting, very recent experiments done by Bates and coworkers ${ }^{15}$ shown that chain length induced fluctuations are more overwhelming for short polymers with $N<$ 100, such strong fluctuations can cause large deviations of order-disorder transition points from the prediction of FH theory. In particular, experimental ODTs are found to be closer to the predictions of particle-based computer simulation models since both experiment and simulations 
have similar finite chain length $N$. These comparisons also confirm the fidelity of the coarse-grained simulations which has more similarity with experimental system.

Variations in the chain length also can cause interesting consequences in polydisperse block copolymer systems where the chain length of the either block has a wide distribution. In the past decades, the effect of polydispersity on the melt phase behavior of long diblock copolymers has been widely investigated both experimentally ${ }^{16}$ and theoretically ${ }^{17}$. Some consistent conclusions have been drawn on the polydispersity effect: it can (i) enlarge the domain spacing of the ordered structures ${ }^{18-20}$ and (ii) consequently shift the OOT boundaries ${ }^{16,17,21,22}$ to a larger volume fraction $(f)$ of minority component; (iii) it can also cause a reduction effect in $(\chi N)$ ODT before $f$ approaching $0.6 .^{16,23}$ The underlying mechanism for the reduction of $(\chi N)$ ODT can be attributed to the accumulation of short blocks at interface region and therefore a reduction of interfacial free energy. ${ }^{20}$ Similarly, a recent Monte Carlo simulation shows that polydispersity of copolymer length or gradient width in synthesized copolymers can be utilized to effectively tune the interfacial properties. ${ }^{24}$ For the two-sided polydisperse system, Matsen ${ }^{19}$ found that the equal polydispersity in both $\mathrm{A}$ and $\mathrm{B}$ blocks will enlarge the domain spacing by a factor of 1.4 when $\mathrm{PDI}\left(\mathrm{PDI} \equiv \mathrm{PDI}_{\mathrm{A}}=\mathrm{PDI}_{\mathrm{B}}\right)$ approaching to 2.0. In the same year, Shi and coworkers ${ }^{21}$ showed that the two-sided polydisperse diblock system still has a symmetric phase diagram, while the stable region for lamella structure was shrank to a smaller size. At the same time, $(\chi N)_{\text {ODT }}$ was found to be decreased more obviously than in the one-sided polydisperse system. Similarly, experimental results ${ }^{25}$ and our simulations ${ }^{26}$ have also shown a strong polydispersity effect in the phase behavior of ABA triblock copolymers, which enhances the stability of a bicontinuous phase in a wide composition range. Real-space self-consistent-field theory study also shown that the polydipersity can influence the vesicle formations from amphiphilic diblock copolymers in solution. ${ }^{27}$ Other than the phase behavior of block copolymers, recent dynamic Monte Carlo simulations also show that chain length polydispersity can also influence the crystal nucleation in rubbers ${ }^{28}$ and the interfacial properties of gradient copolymers. ${ }^{29}$

Although the polydispersity effect has been well studied for conventional long-chain block copolymer systems, there is still a lack of a systematic investigation on the polydispersity effect in short-chain block copolymer systems. As have been discussed in the above, particle-based simulations have a better similarity to experiment due to the short chain length in the system. For instance, for the one-sided polydisperse diblock copolymer system, Lynd and Hillymer's experiment ${ }^{16}$ predicted an obvious up-shift of the ODT boundary when the volume fraction of the polydisperse component exceeds $f=0.6$, however the SCFT work by Matsen ${ }^{17}$ predicted a reduction in $(\chi N)$ ODT over the whole composition range. Instead, particle-based Monte Carlo simulations ${ }^{23}$ showed a very good agreement with experiment. However, Gavrilov et al. ${ }^{30}$ studied the phase behavior for two-sided polydisperse AB diblock copolymers by using DPD simulations. They constructed the phase diagram for the system where both $\mathrm{A}$ and $\mathrm{B}$ blocks are polydisperse with $\mathrm{PDI}_{\mathrm{A}}=\mathrm{PDI}_{\mathrm{B}} \approx 2.0$. Up-shift of the ODT boundaries over the whole composition range ${ }^{30}$ was reported, but the reason was simply attributed to calculation errors. One may wonder if there are more sophisticated explanations? In addition, are there any distinguishable differences between one-sided and two-sided systems?

With the above considerations, we use particle-based dissipative particle dynamics simulation method to investigate the effect of polydispersity on the phase behavior of short diblock copolymer melts. The phase diagrams have been constructed for the monodisperse, one-sided and two-sided polydisperse diblock copolymer systems. The influences of block chain length polydispersity on the changes in ODT boundaries are investigated and the detailed molecular mechanisms are provided. In addition, an unexpected stability of a bicontinuous structure is reported in the case of one-sided polydisperse system.

\section{SIMULATION METHOD AND DETAILS}

Dissipative particle dynamics simulation is a mesoscale simulation technique, which has been well established and tested in the past two decades for the studies of block copolymer phase behaviors ${ }^{31-33}$. In the DPD model, a single particle usually represents a group of atoms or molecules and interparticle interactions are composed of a conservative force $\mathbf{F}^{C}$, a dissipative force $\mathbf{F}^{D}$ and a random force $\mathbf{F}^{R}$, all of these forces are pair-wise. The conservative force is a soft repulsive interaction acting along the connecting line of particle centers:

$$
\mathbf{F}_{i j}^{C}=\left\{\begin{array}{ll}
\alpha_{i j}\left(1-r_{i j} / r_{c}\right) \mathbf{e}_{i j} & \left(r_{i j}<r_{c}\right) \\
0 & \left(r_{i j} \geq r_{c}\right)
\end{array},\right.
$$

where $\alpha_{i j}$ is the maximum repulsion between particle $i$ and $j ; \mathbf{r}_{i j}=\mathbf{r}_{i}-\mathbf{r}_{j}, r_{i j}=\left|\mathbf{r}_{i j}\right|$ and $\mathbf{e}_{i j}=\mathbf{r}_{i j} / r_{i j}$. The cutoff radius, $r_{c}$, is the only length-scale in the system. The dissipative force and random force, serving as the thermostat, are given by:

$$
\begin{aligned}
& \mathbf{F}_{i j}^{D}=-\gamma \omega^{D}\left(r_{i j}\right)\left(\mathbf{v}_{i j} \cdot \mathbf{e}_{i j}\right) \mathbf{e}_{i j}, \\
& \mathbf{F}_{i j}^{R}=\sigma \omega^{R}\left(r_{i j}\right) \xi_{i j} \Delta t^{-1 / 2} \mathbf{e}_{i j},
\end{aligned}
$$

where $\xi_{i j}$ is a random number with zero mean and unit variance, $\mathbf{v}_{i j}$ is the relative velocity between particles. According to the fluctuation-dissipation theorem ${ }^{34}$, the amplitudes of the dissipative force, $\gamma$, and the random force, $\sigma$, as well as r-dependent weight functions, $\omega^{D}$ and $\omega^{R}$, have the relations of $\sigma^{2}=2 \gamma k_{B} T$ and $\omega^{D}\left(r_{i j}\right)=$ $\left[\omega^{R}\left(r_{i j}\right)\right]^{2}$. Following Groot and Warren ${ }^{31}$, a simple form was chosen as:

$$
\omega^{R}\left(r_{i j}\right)=\left\{\begin{array}{ll}
\left(1-r_{i j} / r_{c}\right) & \left(r<r_{c}\right) \\
0 & \left(r \geq r_{c}\right)
\end{array} .\right.
$$


For an easy numerical handling, the cutoff radius $r_{c}$, the particle mass $m$, and the temperature $k_{B} T$ are chosen as the basic units $r_{c}=m=k_{B} T=1$, therefore the unit of time is $\tau=r_{c} \sqrt{m / k_{B} T}=1$.

Polymers are described by the bead-spring model and the adjacent beads in a single polymer chain are tied up by harmonic springs, $\mathbf{F}^{S}=-k_{s} \mathbf{r}_{i j}$, with $k_{s}$ set to 4.0 according to Ref. 32. The time evolution of the interaction particles is governed by Newton's equation of motion. The so-called DPD-VV algorithm is used to propagate the equations of motion with a time step of $\Delta t=0.02$. Each simulation is performed in a simulation box with a size of $40 \times 40 \times 40$ for at least $3 \times 10^{6}$ time steps. This simulation box size is enough to catch the right ODT boundary and the phase behavior of copolymers.

In our simulations, the particle density $\rho$ is set to 3 . The conservative interaction strength $\alpha_{i j}$ can be mapped to Flory-Huggins parameter $\chi$ by $^{32}$ :

$$
\alpha_{i j}=\alpha_{i i}+3.27 \chi \quad(\rho=3),
$$

where $\alpha_{i i}=25$ is the interaction strength between the same type beads. However, as discussed by Groot and Madden $^{32}$, relatively short polymer chain length in DPD simulation comparing with the infinite chain length in theory could artificially increase the amplitude of fluctuations so as to cause unnegligible deviation of the simulated phase diagram from the theory. According to weak coupling calculations of Fredrickson and Helfand ${ }^{13}$, the order-disorder transition at $f=0.5$ with finite chain length shifts as $(\chi N)_{\text {ODT }}=10.5+41.0 N^{-1 / 3}$ for diblock copolymer. At the same time, similar shifts in ODT point is also predicted by Mayes and de la Crutz ${ }^{35}$ to be applicable for block copolymers with any other architectures. Although it is strictly rigorous only in the limit of $N \rightarrow \infty$, a recent viewpoint article by Sinturel, Bates, and Hillmyer ${ }^{36}$ and our previous simulations ${ }^{20,26,37-39}$ demonstrate that a crude application of this relation based on an effective $(\chi N)_{\text {eff parameter is }}$ also applicable even to low $N$ values of 10 as following:

$$
(\chi N)_{\mathrm{eff}}=10.5 \chi N /\left(10.5+41.0 N^{-1 / 3}\right) .
$$

Considering the fact that the short polymers in DPD simulations are swollen, i.e., $R_{g} \sim N^{\nu}$ instead of $R_{g} \sim N^{0.5}$ for non-pertubated Gaussian coils, the mapping relation can be slightly modified as:

$$
\begin{aligned}
(\chi N)_{\mathrm{eff}} & =10.5 \chi N /\left(10.5+41.0 N^{2 / 3-2 \nu}\right) \\
& =\chi N /\left(1+3.9 N^{2 / 3-2 \nu}\right),
\end{aligned}
$$

where $\nu$ is the swelling exponent, which is evaluated by measuring the radius of gyration of polymers as we have done in our previous studies ${ }^{20,37},\left\langle R_{g}^{2}\right\rangle=1 / 6 N^{2 \nu} l^{2}$.

In experiment, $\mathrm{x}$-ray scattering technique is commonly used to determine the mesostructures of block copolymers. For instance, characteristic Bragg reflections ratios $\vec{q} / \vec{q}^{*}$ are at $1,2,3,4,5,6, \cdots$ for a lamellar structure and $1, \sqrt{3}, \sqrt{4}, \sqrt{7}, \sqrt{9}, \sqrt{11}, \cdots$ for a hexagonally packed cylinder structure ${ }^{40}$. In our DPD simulation, the structure factor can be calculated by:

$$
\begin{aligned}
& S(\vec{k})=\rho_{x}(\vec{k}) \rho_{x}(-\vec{k}) / N_{x}, \\
& \rho_{x}(\vec{k})=\sum_{j=1}^{N_{x}} \exp \left(i \vec{k} \cdot{\overrightarrow{r_{j}}}^{x}\right),
\end{aligned}
$$

where $N_{x}$ and the $\rho_{x}(\vec{k})$ are the particle number and the number density of the minority component in the reciprocal space. Wave vector $\vec{q}$ is defined as $\vec{q}=2 \pi / \mathrm{L} * \vec{k}$, $k$ is an integer wave number.

Monodisperse and polydisperse diblock copolymer $\mathrm{A}_{x} \mathrm{~B}_{N_{n}-x}$ melts $\left(f_{\mathrm{A}}=x / N_{n}, f_{\mathrm{B}}=1-f_{\mathrm{A}}\right)$ with average chain length of $N_{n}=10 \sim 12$ are investigated in this study. The molecular weight distribution of polydisperse block follows the well-known Schulz-Zimm distribution $^{41,42}$ as:

$$
p(\sigma)=\frac{n^{n} \sigma^{n-1} \exp (-n \sigma)}{\Gamma(n)}
$$

namely the polydisperse block has a segmental length of $f N_{n} \sigma$, where $\sigma$ is a variable with $\sigma=N / N_{n}$ and polydispersity index PDI is defined as PDI $=(1+\mathrm{n}) / \mathrm{n}$.

\section{RESULTS AND DISCUSSION}

The simulated phase diagrams of monodisperse and polydisperse short diblock copolymer systems are constructed in Fig. 1. The results for monodisperse system with $\mathrm{PDI}_{\mathrm{A}}=\mathrm{PDI}_{\mathrm{B}}=1.00$ are shown in Fig. 1(a) with a comparison with SCFT prediction ${ }^{17}$ (solid lines). The novel lamellae (L), cylinder (C) and gyroid (G) structures obtained in our simulations are well located in the corresponding SCFT phase regions. Spheres are not shown since it's only stable in a relatively narrow composition window and at relatively high segregation strength $\chi N$. In the theoretical ${ }^{17,21}$ predicted compositional window for gyroid structures, we often observe metastable perforated lamellae (PL) instead of gyroid at relatively low segregation strength, shown as solid green circles in the phase diagram. The reason can be attributed to a small difference in the free energies between gyroid and PL phases $^{43}$. To identify the relative stability of G and PL structures, carefully annealing cycles are performed according to Ref. 43. By carefully tuning the simulation box, gyroid structure is obtained at relatively strong segregation strengths, the calculated structure factor and the obtained gyroid structure are plotted in Fig. 2 for illustration. Note that we did not find Fddd ${ }^{44}$ structure formation in our simulation since the phase space for Fddd structure is very narrow. At the symmetrical composition $f=0.5$, the observed $(\chi N)$ ODT is found to be located between $(10.36,10.99)$, the system is in disordered state before $(\chi N)<10.36$ and it is in Lamellae phase when $(\chi N)>10.99$, which is consistent with 


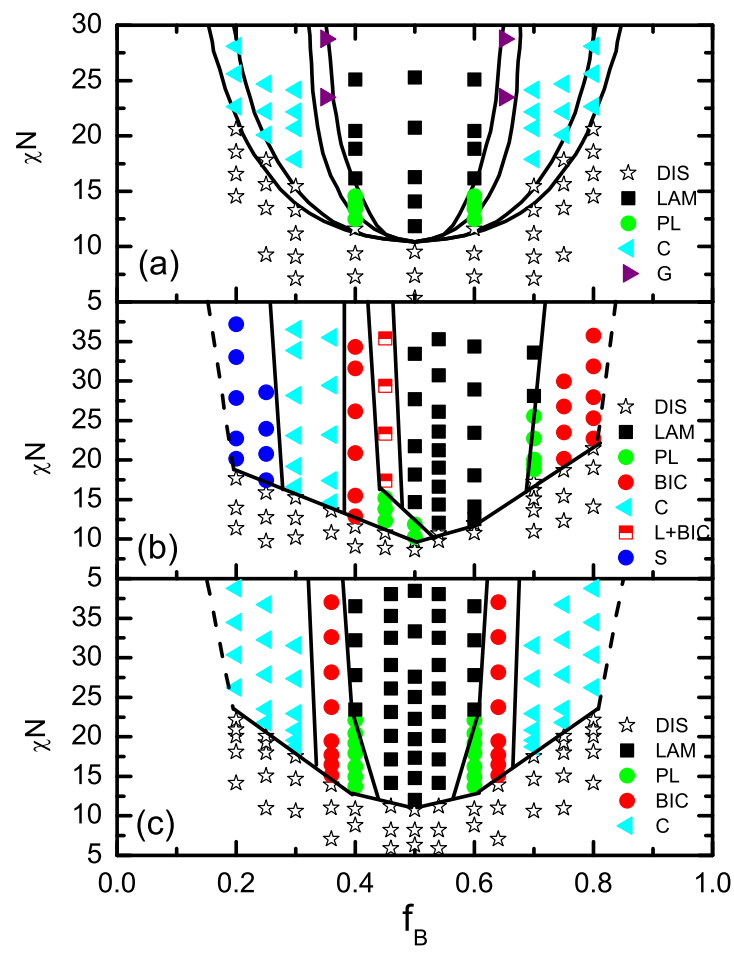

FIG. 1. The simulated phase diagrams of $\mathrm{AB}$ diblock copolymers (a) with monodisperse A and B blocks, (b) with monodisperse A block and polydisperse $\mathrm{B}$ block with $\mathrm{PDI}_{\mathrm{B}}=$ 1.5 , and (c) with polydisperse A block connecting randomly with polydisperse $\mathrm{B}$ block $\left(\mathrm{PDI}_{\mathrm{A}}=\mathrm{PDI}_{\mathrm{B}}=1.5\right)$.

the SCFT or mean-field theory prediction. Overall, we have a nice match with the SCFT results for the whole phase diagram. A small difference lies in the location of the order-order transition boundary between cylinder and spheres. In the SCFT region of spheres with a small $f_{B}$ value, we observed cylinder structures. This might be due to the stretched chain conformation of the short chain model in our simulation while it is Gaussian in SCFT.

The phase diagram of one-sided polydisperse diblock copolymer is demonstrated in Fig. $1(\mathrm{~b})$, where $\mathrm{PDI}_{\mathrm{A}}=$ 1.0 and $\mathrm{PDI}_{\mathrm{B}}=1.5$. With an increase of the volume fraction of polydisperse component $f_{\mathrm{B}}$, regular ordered structures of sphere, cylinder and lamellae can be found in the phase diagram. Due to highly asymmetrical molecular weight distributions in two blocks in the system, the order-order transition (OOT) boundaries are obviously shifted to a higher $f_{\mathrm{B}}$ value. With such shifts in the OOT boundaries, the whole phase diagram becomes highly asymmetric. ${ }^{38} \mathrm{~A}$ direct consequence is the enhancement in the stability of sphere structures formed by polydisperse minority component when $f_{\mathrm{B}}<0.25$, which can be attributed to the long range order broken in cylinder structures induced by the presence of polydispersity. At the same time, the composition window for cylinder phase is squeezed to a smaller region at the left

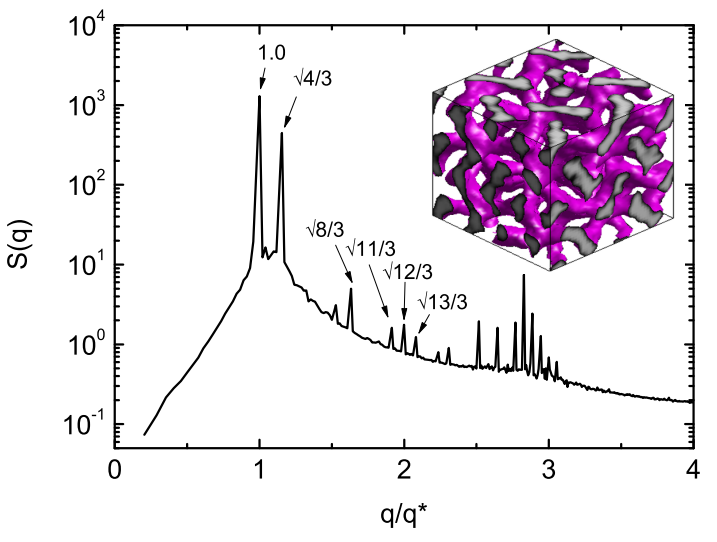

FIG. 2. The calculated structure factor of the gyroid structure (shown in insets) formed in the monodisperse system with $f_{B}=0.35$ at $\chi N=23.47$. The inset shows the gyroid structure obtained in our simulation.

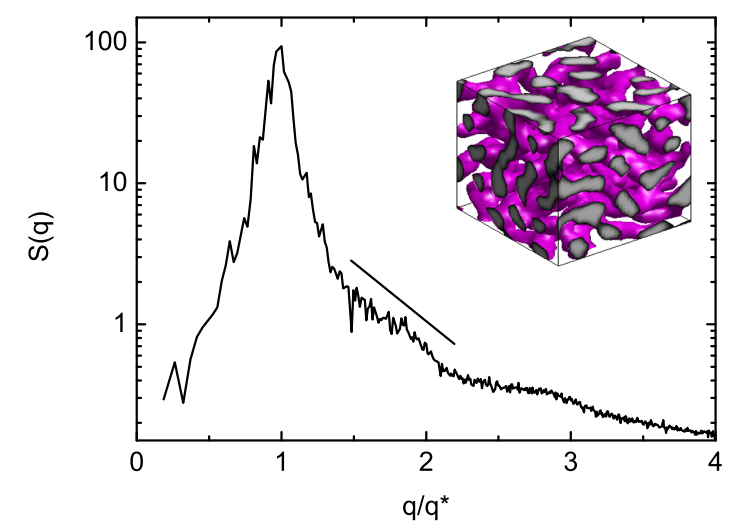

FIG. 3. The calculated structure factor of an irregular bicontinuous structure (shown in insets) formed in one-sided polydisperse system with $f_{B}=0.4$ at $\chi N=30.58$.

side of the phase diagram. We note that at the left side of the phase diagram, a coexistence structure of lamellae and bicontinuous is found before entering the lamellae region. This is also in a good agreement with $\mathrm{SCFT}^{17}$ prediction. A representative isodensity surface structure and its corresponding structure factor is shown in Fig. 4. At the right side of the phase diagram, the cylinder phase disappears when the majority component forming matrix is polydisperse. Instead, an irregular bicontinuous (BIC) phase are observed in the corresponding region. Experimental evidences can be found in polydisperse acrylic diblock copolymers. ${ }^{22}$ Under such a situation, the chains with different chain lengths are difficult to have a homogeneous distribution along the $\mathrm{A} / \mathrm{B}$ interface, leading to a disturbed $\mathrm{A} / \mathrm{B}$ interface and hence formation of a BIC structure with random interconnections forming between cylindrical pipes. A strategy of changing simulation box 


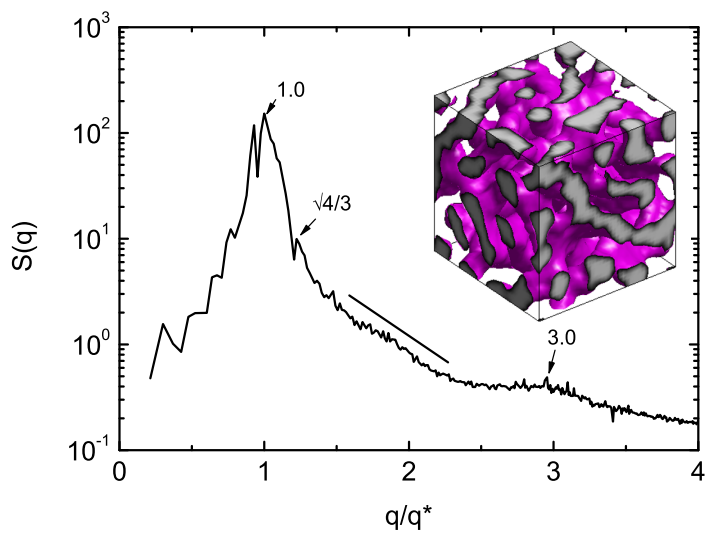

FIG. 4. The calculated structure factor of the coexistence structure (shown in insets) formed in one-sided polydisperse system with $f_{B}=0.46$ at $\chi N=23.32$.

size following Escobedo ${ }^{43}$ was performed to check the stability of the BIC structure. According to $L=2 \pi m / q_{*},{ }^{43}$ an appropriate simulation box size of 34.2 can be estimated. Afterwards, the simulation box size is carefully tuned in the range of 32 to 36 . We find that the BIC structure is very stable in these simulations. However, we note that the BIC structure predicted from our simulation is only expected to be stable with a large enough PDI value. For systems with a small PDI, G structure will be stable in the corresponding region as predicted by SCFT theory. ${ }^{21}$ Recent experiments ${ }^{8,25,45}$ also show that such BIC structure has an unexpected wide stability over $10 \%$ of composition window for ABA type triblock copolymers with a moderate polydispersity in the middle B block. Our previous simulations ${ }^{26}$ even show that BIC structure will be stable over a composition window size of $20 \%$ if both A and B blocks are polydisperse in ABA triblock copolymers. Such bicontinuous structures are desirable for many applications,${ }^{8}$ such as photovoltaic film in solar cells and separator membranes in batteries. In our phase diagram of one-side polydisperse system, this BIC structure is also found to be unexpectedly stable over a composition window size of $10 \%\left(0.7<f_{\mathrm{B}} \leq 0.8\right)$, which has a similar size comparable to that in the case of ABA system. The calculated structure factor using equations 7,8 for the BIC structure obtained in our simulation is plotted in Fig. 1. A characteristic broad scattering peak (a shoulder indicated by a solid line) is observed after the primary peak, which is a typical characteristics for irregular bicontinuous structure, as reported in the experimental BIC structure SAXS pattern ${ }^{25}$. The only difference between two systems is that the BIC structure is formed by polydisperse middle B block in ABA triblock copolymer system with $f_{\mathrm{B}}<0.5$, but by monodisperse A block in $\mathrm{AB}$ diblock copolymer system with $f_{\mathrm{B}}>0.5$. In both cases, B is the polydisperse block. We note that at the left side of the phase diagram, a coexistence structure of lamellae and bicontinuous is found before entering

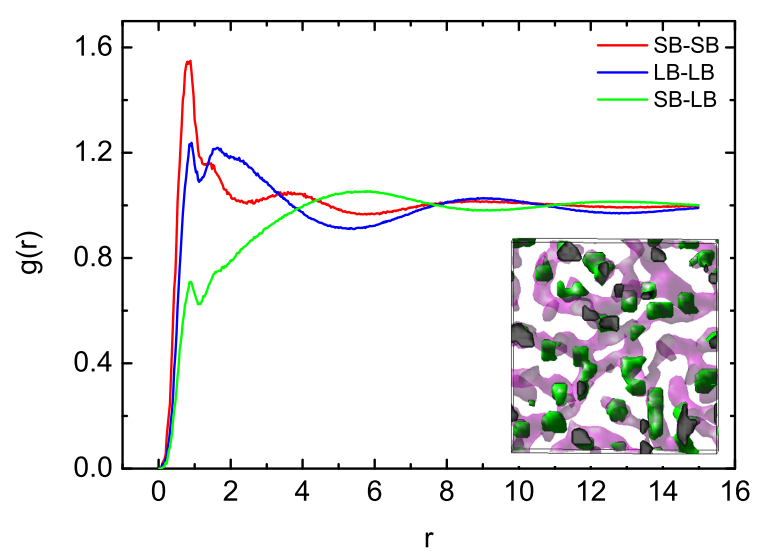

FIG. 5. The radial distribution function between intermolecular B-B pairs in a one-sided polydisperse system at $\chi N_{\text {ODT }} \approx 24$. SB denotes the results for the short B blocks with $N_{\mathrm{B}}=2$ and 3 ; LB is for the long B blocks $N_{\mathrm{B}}=28 \sim$ 45. The inset shows the isodensity surface structure, pink is for all B blocks and green is for LB.

the lamellae region, which is also in a good agreement with SCFT. ${ }^{17}$ A representative isodensity surface structure and its corresponding structure factor is shown in Fig. 4.

Our previous simulations ${ }^{26}$ show that the stabilization mechanism for such BIC structure can be interpreted as the selective spacial distribution of chains with different chain length in space, i.e., long chains are preferably distributed in the branched node structures where has more confrontational space. To further clarify spacial distributions of the chains, we have calculated the radial distribution function, $\mathrm{g}(\mathrm{r})$, between monomer pairs on B blocks with different chain lengths. The results are shown in Fig. 5. The red line indicates the $\mathrm{g}(\mathrm{r})$ function between monomer pairs on short B blocks and the blue line is for $\mathrm{g}(\mathrm{r})$ function between monomer pairs on long $B$ blocks, they are labeled as 'SB-SB' and 'LB-LB' in legend respectively. For the comparison, we also calculated the $\mathrm{g}(\mathrm{r})$ function between short $\mathrm{B}$ and long B block monomers, as shown in green and labeled as 'SB-LB'. Apparently g(r) functions calculated for SB-SB and LBLB pairs have overall a similar intensity. However, much lower intensity of $\mathrm{g}(\mathrm{r})$ function between SB-LB pair at short distances indicates a selective spacial distribution of the long and short chains. The inset shows the isodensity surface for B blocks, the pink is for all B blocks and green is for long B blocks. The results visually show that the long B blocks prefer to be distributed in the nodes of BIC structure where has larger space.

Now, we focus on the two-sided polydisperse AB diblock copolymer system, in which both $\mathrm{A}$ and $\mathrm{B}$ blocks have equal polydispersity $\left(\mathrm{PDI} \equiv \mathrm{PDI}_{A}=\mathrm{PDI}_{B}=1.5\right)$ and both polydisperse $\mathrm{A}$ and $\mathrm{B}$ blocks are connected with each other in a random way to faithfully mimic the sit- 


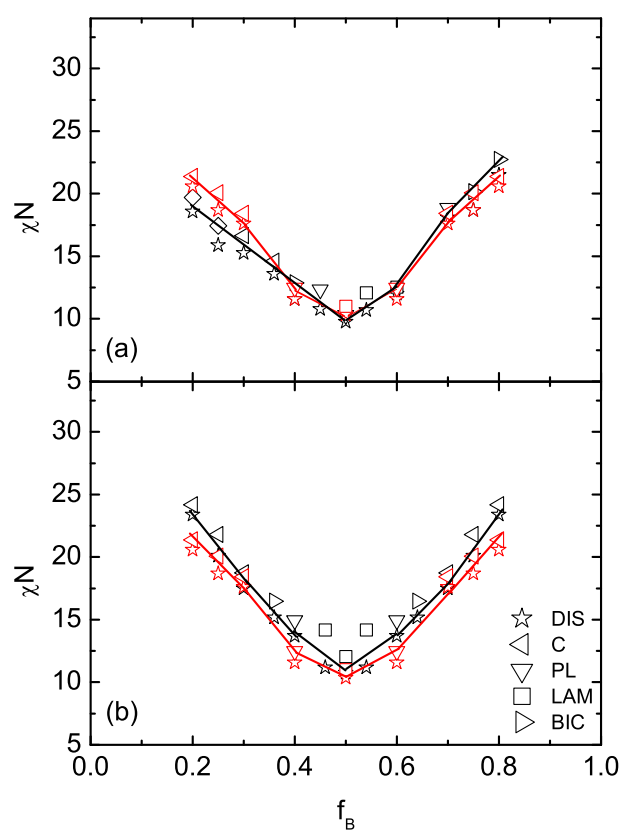

FIG. 6. Comparisons for the ODT boundaries of (a) one-sided and (b) two-sided polydisperse systems with monodisperse system. Black symbols are for polydisperse systems and red ones are for monodisperse system. Solid lines are drawn to guide the eye.

uation in a radical polymerization process. The simulated phase diagram is shown in Fig. 1(c), where the novel ordered lamellar, perforated lamellar, cylindrical and bicontinuous structures are observed as well. We can see that the phase diagram of two-side polydisperse system remains the $f_{A} \leftrightarrow\left(1-f_{A}\right)$ symmetry, which is the same as that of monodisperse system shown in Fig 1(a). The region of lamellar structure shrinks if compared with the monodisperse system, which is also consistent with SCFT study of symmetrical polydisperse diblock copolymer melts by $\mathrm{Shi}^{21}$. However, comparing with the monodisperse system, bicontinuous structure is only stable in a very narrow region.

The above discussions show that the polydispersity in the molecular weight distribution has obvious effects on the stability of ordered structures over different composition windows and consequently on the order-order transition boundaries between these structures. Previous experimental ${ }^{16}$ and theoretical ${ }^{17,21}$ works also show it can influence the order-disorder transition (ODT) boundaries. In order to effectively characterize the ODT boundaries at different $f_{\mathrm{B}}$ values and therefore to illustrate the polydispersity effect on them, systems with different volume fractions of B monomers and polydispersities are simulated at a tightly-spaced $\chi N$ space in the vicinity of the corresponding ODTs. The results for onesided and two-sided polydisperse systems and their comparisons with monodisperse system are shown in Fig. 6, where we only show the disordered/ordered structures

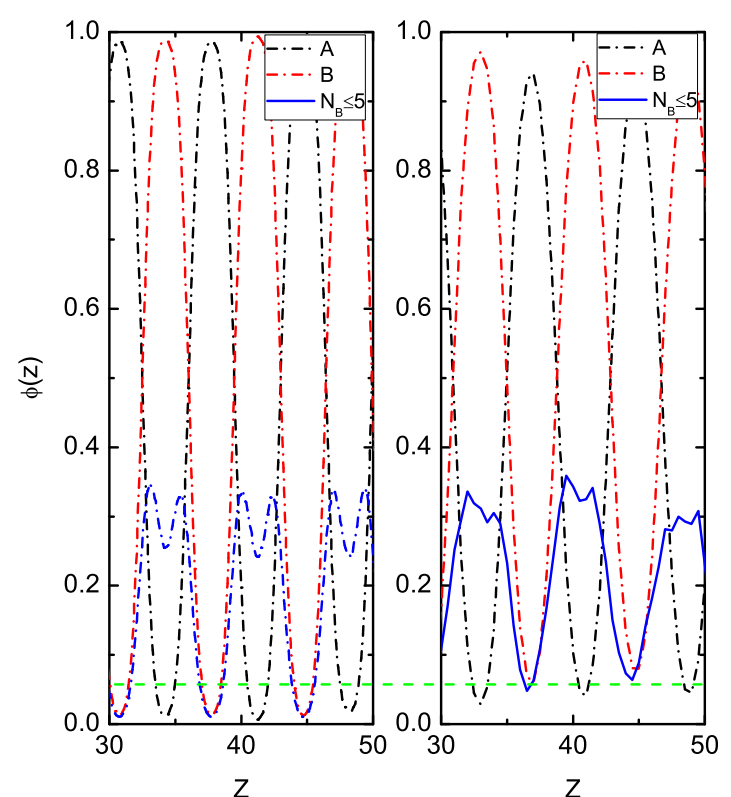

FIG. 7. Density distribution profiles for A and B components in the lamellar structures formed at the segregation strength of $\chi N \approx 18$ (a) in one-sided system with $\mathrm{PDI}_{\mathrm{B}}=1.5$ and (b) in two-sided system with $\mathrm{PDI}_{\mathrm{A}}=\mathrm{PDI}_{\mathrm{B}}=1.5$. Dashed green line is drawn to guide the eye.

exactly before/after ODT. As shown in Fig. 6(a), the deviation of ODT curve in the one-sided polydisperse short diblock copolymer system from the monodisperse system is consistent with the experimental observations ${ }^{16}$ and the Monte Carlo simulations by Matsen ${ }^{23}$, i.e., the $(\chi N)_{\text {ODT }}$ value decreases when the polydisperse block forms the minority component, and it increases when the majority component is polydisperse. The reason can be attributed to the packing frustration release ${ }^{23}$ on the left side of the phase diagram where polydisperse block forms minority component, therefore resulting in a decreasing in $(\chi N)_{\text {ODT }}$. Our previous simulation ${ }^{20}$ also showed that on the left side of the phase diagram, reduction in $(\chi N)$ ODT can also be attributed to an accumulation of short diblocks at the A/B interface and therefore a release in interfacial free energy. Another important fact is that, with the introduction of polydispersity into system, longer polymers will be introduced into system and therefore an domain expansion effect can be found. However, at the right side of the phase diagram the majority component forming matrix are polydisperse and therefore the matrix tends to expand, resulting in a squeezing effect on the structure formed by monodisperse minority A blocks. With such squeezing effect, an increase in interfacial free energy and hence an increase in ODT boundary can be expected. 

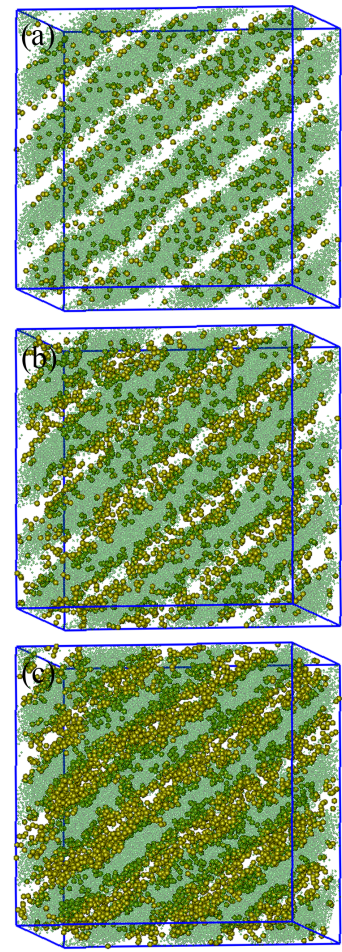

FIG. 8. Visual distributions of (a) $B_{1}$, (b) $B_{2}$, and (c) $B_{3}$ blocks in $A$ lamellar structures formed in two-sided polydisperse system with $\mathrm{PDI}_{\mathrm{A}}=\mathrm{PDI}_{\mathrm{B}}=1.5$ at a segregation strength of $\chi N \approx 18$. The solid yellow beads are $\mathrm{B}$ particles and shadowed green beads are A particles.

In the case of the two-sided polydisperse system, we observe an overall up-shift of the ODT boundary if compared with monodisperse system, as shown in Fig. 6(b). The results are also in consistent with DPD simulations reported in Ref. 30 where the increase in ODT boundary is simply attributed to the calculation errors. However, after a detailed analyze of our results, we show that the up-shift of the ODT boundary is mainly due to a pulling-away effect of short blocks into its opposite domain. First of all, due to the symmetric polydispersity in both A and B blocks, the phase diagram is still symmetric. Considering the polydispersity in both blocks, there are very asymmetric copolymers existing in the system, i.e., with very long $\mathrm{A}$ and very short $\mathrm{B}$ blocks or vice versa. Short A/B blocks in these highly asymmetrical molecules can be easily drawn into the opposite B/A phase by the connecting long B/A blocks. Fig. 7(a) and 7 (b) present the molecule distributions when $\chi N \approx 18$ for one-sided and two-sided polydisperse systems respectively. In Fig. 7(a) for one-sided polydisperse system, the B block with $N_{B} \leq 5$ are distributed almost in the B domain and at the A/B interface. However, in Fig. 7(b), its distribution in the two-sided polydisperse system shows a dramatic pulling-away effect with a certain number of short B blocks pulling away from the B domain into the A domain. This is in consistent with theoretical pre- dictions by Matsen ${ }^{19}$. In addition, such an effect can be doubled due to equal population of the chains which contain very long B and short A blocks in the system. For a more clear illustration of this phenomena, we show in Fig. 8(a), 8(b), 8(c), the visual distributions of B blocks that possesses chain lengths of $N_{B}=1, N_{B}=2$, and $N_{B}=3$, respectively. We can clearly see an obvious distribution of short B blocks with $N_{B}=1$ in the A domain. However, its distribution in A domain decreases very fast even if $N_{B}=2$. With a further increase to $N_{B}=3$, they are mainly distributed at the $\mathrm{A} / \mathrm{B}$ interface as shown in Fig. 8(c). Except the existence of these very asymmetric block copolymers, there are also some molecules with both very short $A$ and $B$ blocks, for instance, $A_{1} B_{1}$. These short molecules are expected to rather homogeneously distributed in the system. Both of the homogeneous distribution of these very short molecules and the distribution of short blocks belonging to very asymmetric molecules in opposite phases can enhance the difficulty in microphase separation, therefore leading to an up-shift in the ODT boundary.

\section{SUMMARY}

Comprehensive dissipative particle dynamics simulations are performed to simulate the phase behavior of monodisperse, one-sided polydisperse, and two-sided polydisperse short diblock copolymers in a $\chi N \sim f$ phase diagram portrait, where $\chi N$ describes segregation strength between different blocks and $f$ is the compositional volume fraction value. By identifying the stability regions for novel lamellae, cylinder, and sphere structures in systems with various designed polydispersities, the dispersity effect on the phase behavior of short diblock copolymer system is investigated in detail. At the same time, the phase diagrams of monodisperse, onesided polydisperse and two-sided polydisperse short AB diblock copolymers are constructed, respectively. Simulated phase diagram for monodisperse system shows a very good agreement with SCFT.

Compared with the phase diagram of monodisperse system, one-sided polydisperse system has an obvious right shift of the order-order transition boundaries to larger content of polydisperse component, which is in a good agreement with SCFT prediction ${ }^{17,21}$. As a consequence, the stabilized composition window for sphere structure is obviously enlarged. Meantime, the cylinder phase disappears at the right part of the phase diagram where the component forming matrix is polydisperse. Very surprisingly, due to a selective spatial distribution of long chains, formation of an irregular bicontinuous phase is found in the composition region where formation of the cylinder phase is expected for the monodisperse system. Such bicontinuous structure is very similar to the one found in polydisperse ABA type triblock copolymer systems. ${ }^{25,26}$ In addition, with a very good agreement with the experimental observation by Lynd 
and Hillmyer, ${ }^{16}$ a reduction in the order-disorder transition boundary $(\chi N)_{\text {ODT }}$ in the left side of the phase diagram with $f_{B}<0.5$ and an increase in $(\chi N)_{\text {ODT }}$ in the right side of the phase diagram with $f_{B}>0.5$ are found, where $f_{B}$ is the volume fraction of the polydisperse B block. At the left side of the phase diagram, the reduction in $(\chi N)$ ODT can be attributed to the release of packing frustration and a decrease in interfacial free energy due to accumulation of short compatibilizer chains at the interface. However, at the right side of the phase diagram, the matrix component is polydisperse and expansion in the matrix will undoubtedly cause an squeezing effect on the domain therefore will increase the interfacial free energy and hence up-shift the $(\chi N)_{\text {ODT }}$ value.

In the two-sided polydisperse system, the phase diagram has a very good symmetry around $f=0.5$ due to symmetrical chain length distribution in two blocks. Overall, the phase diagram is very similar to the one for monodisperse system, where we can find the formations of cylinder, (perforated) lamellae and the irregular bicontinuous structures. Comparing with monodisperse system, a major difference lies in the position of the order-disorder transition boundary. For which an obvious up-shift to larger $(\chi N)$ ODT values is observed at all compositions we studied. This is found to be related to the presence of highly asymmetric chains in the system. Very short A blocks in these asymmetric chains can be pulled away from its corresponding domain and into the opposite domain by connecting long B blocks and vice versa. Such pulling-away phenomena will cause an interruptive effect for forming ordered structures, leading to an increase in $(\chi N)_{\mathrm{ODT}}$.

\section{ACKNOWLEDGEMENT}

This work is supported by the National Science Foundation of China (21522401, 21374043, 21204029), and subsidized by the National Basic Research Program of China (973 Program, 2012CB821500). H.J.Q. and Z.Y.L. are also thankful for the support of the Jilin Province Science and Technology Development Plan (20130101020JC, 20140519004JH).

${ }^{1}$ F. S. Bates and G. H. Fredrickson, Phys. Today 52, 32 (1999).

${ }^{2}$ G. Liu, I. Wyman, Sci. China Chem. 56, 1040 (2013).

${ }^{3}$ C.-I. Huang, React. Funct. Polym. 69, 530 (2009).

${ }^{4}$ C.-I. Huang and L.-F. Yang, Macromolecules 43, 9117 (2010).

${ }^{5}$ B. Yu, J. Deng, B. Li, and A.-C. Shi, Soft Matter 10, 6831 (2014).

${ }^{6}$ A.-C. Shi and B. Li, Soft Matter 9, 1398 (2013).

${ }^{7}$ X. Cao, L. Zhang, J. Gu, L. Wang, J. Lin, Polymer 72, 10 (2015).
${ }^{8}$ R. A. Register, Nature 483, 166 (2012).

${ }^{9}$ M. P. Stoykovich and P. F. Nealey, Mater. Today 9, 20 (2006).

${ }^{10}$ Y. Luo, D. Montarnal, S. Kim, W. Shi, K. P. Barteau, C. W. Pester, P. D. Hustad, M. D. Christianson, G. H. Fredrickson, E. J. Kramer, and C. J. Hawker, Macromolecules 48, 3422 (2015).

${ }^{11}$ S. Lee, T. M. Gillard, F. S. Bates, AIChE J 59, 3502 (2013).

${ }^{12} \mathrm{M}$. W. Matsen, Macromolecules 45, 8502 (2012).

${ }^{13}$ G. H. Fredrickson and E. Helfand, J. Chem. Phys. 87, 697 (1987).

${ }^{14}$ J. Glaser, P. Medapuram, T. M. Beardsley, M. W. Matsen, and D. C. Morse, Phys. Rev. Lett. 113, 068302 (2014).

${ }^{15}$ T. M. Gillard, P. Medapuram, D. C. Morse, and F. S. Bates, Macromolecules 48, 2801 (2015).

${ }^{16}$ N. A. Lynd and M. A. Hillmyer, Macromolecules 40, 8050 (2007).

${ }^{17}$ M. Matsen, Phys. Rev. Lett. 99, 148304 (2007).

${ }^{18}$ N. A. Lynd and M. A. Hillmyer, Macromolecules 38, 8803 (2005). ${ }^{19}$ M. W. Matsen, Eur. Phys. J. E 21, 199 (2006).

${ }^{20}$ Y. Li, H.-J. Qian, and Z.-Y. Lu, Polymer 54, 3716 (2013).

${ }^{21}$ D. M. Cooke and A.-C. Shi, Macromolecules 39, 6661 (2006).

${ }^{22}$ A.-V. Ruzette, S. Tenc-Girault, L. Leibler, F. Chauvin, D. Bertin, O. Guerret, and P. Grard, Macromolecules 39, 5804 (2006).

${ }^{23}$ T. M. Beardsley and M. W. Matsen, Macromolecules 44, 6209 (2011).

${ }^{24}$ D. Sun and H. Guo, Polymer 63, 82 (2015).

${ }^{25}$ J. M. Widin, A. K. Schmitt, A. L. Schmitt, K. Im, and M. K. Mahanthappa, J. Am. Chem. Soc. 134, 3834 (2012).

${ }^{26}$ Y. Li, H.-J. Qian, Z.-Y. Lu, and A.-C. Shi, Polymer 54, 6253 (2013).

${ }^{27}$ Y. Jiang, T. Chen, F. Ye, H. Liang, and A.-C. Shi, Macromolecules 38, 6710 (2005).

${ }^{28}$ M.-M. Zhang, L.-Y. Zha, H.-H. Gao, Y.-J. Nie, and W.-B. Hu, Chi. J. Polym. Sci. 32, 1218 (2014).

${ }^{29}$ D. Sun and H. Guo, Polymer 63, 82 (2015).

${ }^{30}$ A. A. Gavrilov, Y. V. Kudryavtsev, and A. V. Chertovich, J. Chem. Phys. 139, 224901 (2013).

${ }^{31}$ R. D. Groot and P. B. Warren, J. Chem. Phys. 107, 4423 (1997).

${ }^{32}$ R. D. Groot and T. J. Madden, J. Chem. Phys. 108, 8713 (1998).

${ }^{33}$ H.-J. Qian, Z.-Y. Lu, L.-J. Chen, Z.-S. Li, and C.-C. Sun, J. Chem. Phys. 122, 184907 (2005).

${ }^{34}$ P. Espanol and P. Warren, Eur. Phys. Lett. 30, 191 (1995).

${ }^{35}$ A. M. Mayes and M. Olvera de la Cruz, J. Chem. Phys. 95, 4670 (1991).

${ }^{36}$ C. Sinturel, F. S. Bates, and M. A. Hillmyer, ACS Macro Lett. 4, 1044 (2015).

${ }^{37}$ H.-J. Qian, Z.-Y. Lu, L.-J. Chen, Z.-S. Li, and C.-C. Sun, Macromolecules 38, 1395 (2005).

${ }^{38}$ Y. Li, H.-J. Qian, Z.-Y. Lu, and A.-C. Shi, J. Chem. Phys. 139, 096101 (2013).

${ }^{39}$ Y. Li, H.-J. Qian, and Z.-Y. Lu, Eur. Phys. Lett. 103, 68001 (2013).

${ }^{40}$ A. J. Ryan, S.-M. Mai, J. Patrick A. Fairclough, I. W. Hamley, and C. Booth, Phys. Chem. Chem. Phys. 3, 2961 (2001).

${ }^{41}$ B. H. Zimm, J. Chem. Phys. 16, 1099 (1948).

${ }^{42}$ N. A. Lynd, M. A. Hillmyer, and M. W. Matsen, Macromolecules 41, 4531 (2008).

${ }^{43}$ F. J. Martinez-Veracoechea and F. A. Escobedo, J. Chem. Phys. 125, 104907 (2006).

${ }^{44}$ M. Takenaka, T. Wakada, S. Akasaka, S. Nishitsuji, K. Saijo, H. Shimizu, M. I. Kim, and H. Hasegawa, Macromolecules 40, 4399 (2007).

${ }^{45}$ J. M. Widin, A. K. Schmitt, K. Im, A. L. Schmitt, and M. K. Mahanthappa, Macromolecules 43, 7913 (2010). 
